\title{
ENHIDE telehealth primary care support of adults with diabetes and chronic kidney disease
}

\author{
Authors: Dawn Hardy, Peter Winocour, Karen Moore-Haines, Anne Currie, Andrew Solomon and Claire Renshaw
}

\begin{abstract}
Aims
Diabetes and chronic kidney disease (CKD) commonly coexist and management is complex with frequent cardiovascular, retinal, foot and bone multi-morbidity, and increased risks of hospitalisation. The East and North Hertfordshire Clinical Commissioning Group (CCG) commissioned a pilot service to help identify and then provide a holistic virtual review of high-risk patients with diabetes and CKD, by specialist diabetes consultants, followed by Skype case-based discussions with general practitioner (GP) practices, providing an opportunity to educate and upskill primary care colleagues to help manage vulnerable patients with type 2 diabetes and CKD. In addition the project was designed to recognise the large burden of unmet clinical need and the priority to develop alternatives to standard outpatient- based specialist support of primary care for complex long-term conditions.
\end{abstract}

\section{Methods}

The team initially collaborated with the information technology department of the CCG to obtain data from SystmOne and EMIS, with the majority of the 55 general practices $(n=43)$ using the SystmOne clinical management system. We selected four pilot practices to test patient data acquisition and refine the process. All 55 GP surgeries were invited to participate in a systematic review of patients with CKD on their diabetes registers with estimated glomerular filtration rate (eGFR) $<60$ and/or urine albumin:creatinine ratios $\geq 10$. Where participation was low in locality areas, practices were randomly selected to ensure a balanced representative geographical and socio-economical split, with varying deprivation indices within east and north Hertfordshire. All patient data was analysed and individual case advice was produced by the project consultant diabetologists and conveyed to the practice.

In addition, a Skype-based consultation was arranged with the primary care team where case records of 20 patients were identified for a focused review to facilitate active discussion, teaching and education. All identified patients were enabled to have access to a diabetes 'sick day rule' card to help manage acute illness with risk of acute or chronic kidney injury through temporary withdrawal of potentially nephrotoxic medication and appropriate management of glycaemia.

\section{Results}

To date, 15 surgeries have provided information on 2,091 people with diabetes and CKD who have undergone virtual case review, representing on average $23 \%$ (range $10-28 \%$ ) of individuals on the general practice diabetes registers. On average, $82 \%$ of these were solely managed in primary care with the remainder under specialist diabetes and/or renal hospital clinics.

Of these $2,091,10 \%$ required referral for specialist diabetes care, $14 \%$ assessment for significant deterioration in renal function, $25 \%$ for medication change for blood pressure, $38 \%$ for medication change for lipid management, $10 \%$ for initiation of antiplatelet therapy, 25\% for change of glycaemic-lowering therapy, $35 \%$ for assessment of metabolic bone health and $29 \%$ who required advice input regarding high risk for neurovascular foot complications. Anaemia (haemoglobin $<110 \mathrm{~g} / \mathrm{L}$ ) was present in $13 \%$ and predominantly in those with CKD over the age of 70. Currently five practices have had Skype telehealth case reviews and all have returned extremely positive feedback regarding the process.

\section{Conclusion}

This unique model has demonstrated that it is possible for specialist physicians to work with primary care more closely to evaluate large numbers of complex patients virtually, and to use alternatives to traditional outpatient consultations to provide consultant advice. Furthermore the project to date has uncovered a large burden of unrecognised unmet clinical need in a vulnerable cohort. This model of care could have wide applicability for a range of other complex long-term conditions.

\section{Conflict of interest statement}

There are no conflicts of interest. 